Kragujevac Journal of Mathematics

Volume 41(2) (2017), Pages 167-178.

\title{
POSITIVE SOLUTIONS FOR A SECOND-ORDER DIFFERENCE EQUATION WITH SUMMATION BOUNDARY CONDITIONS
}

\author{
F. BOUCHELAGHEM ${ }^{1}$, A. ARDJOUNI ${ }^{2}$, AND A. DJOUDI $^{1}$
}

\begin{abstract}
In this article we study the existence of positive solutions for a secondorder difference equation with summation boundary conditions. The main tool employed here is the Krasnoselskii's fixed point theorem in a cone.
\end{abstract}

\section{INTRODUCTION}

The study of the existence of solutions of multipoint boundary value problems for linear second order ordinary differential and difference equations was initiated by Ilin and Moiseev [8]. Then Gupta [5] studied three-point boundary value problems for nonlinear second order ordinary differential equations. Since then, the existence of positive solutions for nonlinear second-order three point boundary value problems have also been studied by many authors by using the fixed point theorems or coincidence degree theory, one may see the text books $[1,2]$ and the papers $[3-7,10-17]$.

Liu [12] proved the existence of single and multiple positive solutions for the threepoint boundary value problem

$$
\begin{cases}u^{\prime \prime}(t)+a(t) f(u(t))=0, & t \in(0,1), \\ u^{\prime}(0)=0, & u(1)=\beta u(\eta),\end{cases}
$$

where $0<\eta<1$ and $0<\beta<1$.

Key words and phrases. Positive solutions, Krasnoselskii's fixed point theorem, difference equations, three-point summation boundary value problems, cones.

2010 Mathematics Subject Classification. Primary: 39A05. Secondary: 39A10, 39A12.

Received: January 27, 2016.

Accepted: August 31, 2016. 
In [14], Sitthiwirattham and Ratanapun considered the following three-point summation boundary value problem

$$
\begin{cases}\triangle^{2} u(t-1)+a(t) f(u(t))=0, & t \in\{1,2, \ldots, T\}, \\ u(0)=0, & u(T+1)=\alpha \sum_{s=1}^{\eta} u(s),\end{cases}
$$

where $f$ is continuous, $T \geq 3$ is a fixed positive integer, $\eta \in\{1,2, \ldots, T-1\}, 0<\alpha<$ $\frac{2 T+2}{\eta(\eta+1)}$. They obtained the existence of positive solutions by using the Krasnoselskii's fixed point theorem in cones.

Chasreechai and Sitthiwirattham [4] proved the existence of solutions for the boundary value problem with summation condition

$$
\begin{cases}\triangle^{2} u(t-1)+f(t, u(t))=0, & t \in\{1,2, \ldots, T\}, \\ u(0)=0, & u(T+1)=\alpha \sum_{s=1}^{\eta} u(s),\end{cases}
$$

where $\frac{2 T+2}{\alpha \eta(\eta+1)}=1, T \geq 3, \eta \in\{1,2, \ldots, T-1\}$ and $f$ is continuous function.

In [13], Reunsumrit and Sitthiwirattham investigated the existence of positive solutions for the boundary value problem with summation condition

$$
\begin{cases}\triangle^{2} u(t-1)+f(t, u(t))=0, & t \in\{1,2, \ldots, T\}, \\ u(0)=\beta \triangle u(0), & u(T+1)=\alpha \sum_{s=1}^{\eta} u(s),\end{cases}
$$

where $f$ is continuous, $T \geq 3, \eta \in\{1,2, \ldots, T-1\}$ and

$$
0<\alpha<\frac{2 T+2}{\eta(\eta+1)}, \quad 0<\beta<\frac{2 T+2-\alpha \eta(\eta+1)}{2(\alpha \eta-1)} .
$$

By employing the Krasnoselskii fixed point theorem, the authors obtained the positivity results.

The boundary value problem with summation conditions

$$
\begin{cases}\triangle^{2} u(t-1)+a(t) f(u(t))=0, & t \in\{1,2, \ldots, T\}, \\ u(0)=\beta \sum_{s=1}^{\eta} u(s), & u(T+1)=\alpha \sum_{s=1}^{\eta} u(s),\end{cases}
$$

has been investigated in [15], where $f$ is continuous, $T \geq 3, \eta \in\{1,2, \ldots, T-1\}$ and

$$
0<\alpha<\frac{2 T+2}{\eta(\eta+1)}, \quad 0<\beta<\frac{2 T+2-\alpha \eta(\eta+1)}{\eta(2 T-\eta+1)} .
$$

By using the Krasnoselskii's fixed point theorem, the existence of positive solutions has been established.

In this paper, we are interested in the analysis of qualitative theory of the problems of the existence of positive solutions to second-order difference equations. Inspired and motivated by the works mentioned above and the papers $[3-7,10-17]$ and the references 
therein, we concentrate on the existence of positive solutions for the following secondorder difference equation with three-point summation boundary value problem

$$
\begin{cases}\triangle^{2} u(t-1)+a(t) f(u(t))=0, & t \in\{1,2, \ldots, T\}, \\ \triangle u(0)=0, & u(T+1)=\alpha \sum_{s=1}^{\eta} u(s),\end{cases}
$$

where $f$ is continuous, $T \geq 3$ is a fixed positive integer, $\eta \in\{1,2, \ldots, T-1\}$.

The aim of this paper is to give some results for existence of positive solutions to (1.1), assuming that $0<\alpha<\frac{1}{\eta}$ and $f$ is either superlinear or sublinear. Set

$$
f_{0}=\lim _{u \rightarrow 0^{+}} \frac{f(u)}{u}, \quad f_{\infty}=\lim _{u \rightarrow \infty} \frac{f(u)}{u} .
$$

Then $f_{0}=0$ and $f_{\infty}=\infty$ correspond to the superlinear case, and $f_{0}=\infty$ and $f_{\infty}=0$ correspond to the sublinear case.

Let $\mathbb{N}$ be the non negative integer, we let $\mathbb{N}_{i, j}=\{k \in \mathbb{N}: i \leq k \leq j\}$ and $\mathbb{N}_{p}=\mathbb{N}_{0, p}$. By the positive solution of (1.1), we mean that a sequence $u: \mathbb{N}_{T+1} \rightarrow(0, \infty)$ and satisfies the problem (1.1).

Throughout this paper, we suppose the following conditions hold:

(A1) $f \in C([0, \infty),[0, \infty))$,

(A2) $a(t) \geq 0$ for all $t \in \mathbb{N}_{1, T}$ and there exists $t_{0} \in \mathbb{N}_{\eta, T}$ such that $a\left(t_{0}\right)>0$.

The proof of the main theorem is based upon an application of the following Krasnoselskii's fixed point theorem in a cone.

Theorem 1.1 ([9]). Let $E$ be a Banach space, and let $K \subset E$ be a cone. Assume $\Omega_{1}$, $\Omega_{2}$ are open subsets of $E$ with $0 \in \Omega_{1}, \bar{\Omega}_{1} \subset \Omega_{2}$, and let

$$
A: K \cap\left(\bar{\Omega}_{2} \backslash \Omega_{1}\right) \rightarrow K,
$$

be a completely continuous operator such that:

(i) $\|A u\| \leq\|u\|, u \in K \cap \partial \Omega_{1}$, and $\|A u\| \geq\|u\|, u \in K \cap \partial \Omega_{2}$,

(ii) or $\|A u\| \geq\|u\|, u \in K \cap \partial \Omega_{1}$, and $\|A u\| \leq\|u\|, u \in K \cap \partial \Omega_{2}$.

Then $A$ has a fixed point in $K \cap\left(\bar{\Omega}_{2} \backslash \Omega_{1}\right)$.

\section{PRELIMINARIES}

We now state and prove several lemmas before stating our main results.

Lemma 2.1. Let $\alpha \eta \neq 1$. Then, for $y \in \mathbb{R}^{T}$, the problem

$$
\begin{cases}\triangle^{2} u(t-1)+y(t)=0, & t \in \mathbb{N}_{1, T}, \\ \triangle u(0)=0, & u(T+1)=\alpha \sum_{s=1}^{\eta} u(s),\end{cases}
$$


has a unique solution

$$
\begin{aligned}
u(t)= & \frac{1}{1-\alpha \eta} \sum_{s=1}^{T}(T-s+1) y(s)-\frac{\alpha}{2(1-\alpha \eta)} \sum_{s=1}^{\eta-1}(\eta-s)(\eta-s+1) y(s) \\
& -\sum_{s=1}^{t-1}(t-s) y(s), t \in \mathbb{N}_{T+1} .
\end{aligned}
$$

Proof. From (2.1), we get

$$
\begin{aligned}
\triangle u(t)-\triangle u(t-1) & =-y(t) \\
\triangle u(t-1)-\triangle u(t-2) & =-y(t-1) \\
& \vdots \\
\triangle u(1)-\triangle u(0) & =-y(1) .
\end{aligned}
$$

We sum the above equations to obtain

$$
\triangle u(t)=\triangle u(0)-\sum_{s=1}^{t} y(s), \quad t \in \mathbb{N}_{T}
$$

from $\triangle u(0)=0$, we have

$$
\triangle u(t)=-\sum_{s=1}^{t} y(s), \quad t \in \mathbb{N}_{T}
$$

We denote $\sum_{s=p}^{q} y(s)=0$, if $p>q$. Similarly, we sum (2.2) from $t=0$ to $t=h$, we get

$$
u(h+1)=u(0)-\sum_{s=1}^{h}(h+1-s) y(s), \quad h \in \mathbb{N}_{T},
$$

by changing the variable from $h+1$ to $t$, we have

$$
u(t)=u(0)-\sum_{s=1}^{t-1}(t-s) y(s), \quad t \in \mathbb{N}_{T+1} .
$$

We $\operatorname{sum}(2.3)$ from $t=1$ to $t=\eta$, we obtain

$$
\sum_{s=1}^{\eta} u(s)=\eta u(0)-\sum_{s=1}^{\eta-1} \sum_{l=1}^{\eta-s} l y(s)=\eta u(0)-\frac{1}{2} \sum_{s=1}^{\eta-1}(\eta-s)(\eta-s+1) y(s) .
$$

By (2.3) from $u(T+1)=\alpha \sum_{s=1}^{\eta} u(s)$, we get

$$
(1-\alpha \eta) u(0)=\sum_{s=1}^{T}(T-s+1) y(s)-\frac{\alpha}{2} \sum_{s=1}^{\eta-1}(\eta-s)(\eta-s+1) y(s) .
$$


Therefore,

$$
u(0)=\frac{1}{1-\alpha \eta} \sum_{s=1}^{T}(T-s+1) y(s)-\frac{\alpha}{2(1-\alpha \eta)} \sum_{s=1}^{\eta-1}(\eta-s)(\eta-s+1) y(s) .
$$

Hence, (2.1) has a unique solution

$$
\begin{aligned}
u(t)= & \frac{1}{1-\alpha \eta} \sum_{s=1}^{T}(T-s+1) y(s)-\frac{\alpha}{2(1-\alpha \eta)} \sum_{s=1}^{\eta-1}(\eta-s)(\eta-s+1) y(s) \\
& -\sum_{s=1}^{t-1}(t-s) y(s), t \in \mathbb{N}_{T+1} .
\end{aligned}
$$

Lemma 2.2. Let $0<\alpha<\frac{1}{\eta}$. If $y(t) \geq 0$ for all $t \in \mathbb{N}_{1, T}$, then the unique solution $u$ of (2.1) satisfies $u(t) \geq 0$ for $t \in \mathbb{N}_{T+1}$.

Proof. From the fact that $\triangle^{2} u(t-1)=u(t+1)-2 u(t)+u(t-1)=-y(t) \leq 0$, we know that $\triangle u(t)$ is a monotone decreasing sequence. Thus $\triangle u(t) \leq \triangle u(0)=0$ and $u$ is a monotone decreasing sequence, this is $u(t) \geq u(T+1)$. So, if $u(T+1) \geq 0$, then $u(t) \geq 0$ for $t \in \mathbb{N}_{T+1}$.

If $u(T+1)<0$, then $\sum_{s=1}^{\eta} u(s)<0$. Since $\sum_{s=1}^{\eta} u(s) \geq \eta u(\eta)$, we get

$$
u(T+1)=\alpha \sum_{s=1}^{\eta} u(s)>\frac{1}{\eta} \sum_{s=1}^{\eta} u(s) \geq u(\eta)
$$

that is

$$
u(T+1)>u(\eta)
$$

which contradicts the fact that $u$ is a monotone decreasing sequence.

Lemma 2.3. Let $\alpha>\frac{1}{\eta}$. If $y(t) \geq 0$ for all $t \in \mathbb{N}_{1, T}$, then (2.1) has no positive solution.

Proof. Assume (2.1) has a positive solution $u$.

If $u(T+1)>0$, then $\sum_{s=1}^{\eta} u(s)>0$. It implies

$$
u(T+1)=\alpha \sum_{s=1}^{\eta} u(s)>u(\eta)
$$

that is

$$
u(T+1)>u(\eta)
$$

which is a contradiction to the fact that $u$ is a monotone decreasing sequence.

If $u(T+1)=0$, then $\sum_{s=1}^{\eta} u(s)=0$, this $u(t)=0$ for all $t \in \mathbb{N}_{\eta}$. If there exists $t_{0} \in \mathbb{N}_{\eta+1, T}$ such that $u\left(t_{0}\right)>0$, the $u(0)=u(\eta)<u\left(t_{0}\right)$, a contradiction with the fact that $u$ is a monotone decreasing sequence. Therefore, no positive solutions exist. 
In the rest of the paper, we assume that $0<\alpha<\frac{1}{\eta}$. Moreover, we will work in the Banach space $\mathbb{R}^{T+2}$, and only the sup norm is used.

Lemma 2.4. Let $0<\alpha<\frac{1}{\eta}$. If $y(t) \geq 0$ for all $t \in \mathbb{N}_{1, T}$, then the unique solution $u$ of (2.1) satisfies

$$
\min _{t \in \mathbb{N}_{T+1}} u(t) \geq \gamma\|u\|
$$

where

$$
\gamma=\frac{\alpha \eta(T+1-\eta)}{T+1-\alpha \eta^{2}}
$$

Proof. By Lemma 2.2, we know that

$$
u(T+1) \leq u(t) \leq u(0) .
$$

So

$$
\min _{t \in \mathbb{N}_{T+1}} u(t)=u(T+1), \quad \max _{t \in \mathbb{N}_{T+1}} u(t)=u(0) .
$$

From the fact that $u$ is monotone decreasing, we get

$$
u(T+1)=\alpha \sum_{s=1}^{\eta} u(s) \geq \alpha \eta u(\eta) .
$$

Since $\Delta^{2} u(t) \leq 0$ and $\Delta u(t) \leq 0$ for $t \in \mathbb{N}_{T+1}$, we have

$$
\frac{u(0)-u(T+1)}{-(T+1)} \geq \frac{u(T+1)-u(\eta)}{(T+1)-\eta} .
$$

By (2.6), we get

$$
\begin{aligned}
u(0) & \leq u(T+1)-\frac{u(T+1)-u(\eta)}{(T+1)-\eta}(T+1) \\
& \leq u(T+1)\left(1-\frac{1-\frac{1}{\alpha \eta}}{T+1-\eta}(T+1)\right) \\
& =u(T+1) \frac{T+1-\alpha \eta^{2}}{\alpha \eta(T+1-\eta)} .
\end{aligned}
$$

Combining this with (2.5), we obtain

$$
\min _{t \in \mathbb{N}_{T+1}} u(t) \geq \frac{\alpha \eta(T+1-\eta)}{T+1-\alpha \eta^{2}}\|u\| .
$$

\section{Main Results}

Now we are in the position to establish the main results.

Theorem 3.1. Assume (A1) and (A2) hold. Then the problem (1.1) has at least one positive solution in the case

(i) $f_{0}=0$ and $f_{\infty}=\infty$ (superlinear), 
(ii) or $f_{0}=\infty$ and $f_{\infty}=0$ (sublinear).

Proof. It is known that $0<\alpha<\frac{1}{\eta}$. From Lemma 2.1, $u$ is a solution to the boundary value problem (1.1) if and only if $u$ is a fixed point of operator $A$, where $A$ is defined by

$$
\begin{aligned}
u(t)= & \frac{1}{1-\alpha \eta} \sum_{s=1}^{T}(T-s+1) a(s) f(u(s)) \\
& -\frac{\alpha}{2(1-\alpha \eta)} \sum_{s=1}^{\eta-1}(\eta-s)(\eta-s+1) a(s) f(u(s))-\sum_{s=1}^{t-1}(t-s) a(s) f(u(s)) \\
:= & (A u)(t) .
\end{aligned}
$$

Denote

$$
K=\left\{u \in \mathbb{R}^{T+2}, u(t) \geq 0 \text { for all } t \in \mathbb{N}_{T+1}, \min _{t \in \mathbb{N}_{T+1}} u(t) \geq \gamma\|u\|\right\} .
$$

where $\gamma$ is defined in (2.4).

It is obvious that $K$ is a cone in $\mathbb{R}^{T+2}$. By Lemma $2.4, A(K) \subseteq K$. It is also easy to check that $A: K \rightarrow K$ is completely continuous.

(i) Superlinear case: $f_{0}=0$ and $f_{\infty}=\infty$. Since $f_{0}=0$, we may choose $L_{1}>0$ so that $f(u) \leq \varepsilon u$, for $0<u \leq L_{1}$, where $\varepsilon>0$ satisfies

$$
\frac{\varepsilon}{1-\alpha \eta} \sum_{s=1}^{T}(T-s+1) a(s) \leq 1 .
$$

Thus, if we let

$$
\Omega_{1}=\left\{u \in \mathbb{R}^{T+2}, u(t) \geq 0 \text { for all } t \in \mathbb{N}_{T+1},\|u\|<L_{1}\right\},
$$

then for $u \in K \cap \partial \Omega_{1}$, we get

$$
\begin{aligned}
A u(t) & \leq \frac{1}{1-\alpha \eta} \sum_{s=1}^{T}(T-s+1) a(s) f(u(s)) \\
& \leq \frac{\varepsilon}{1-\alpha \eta} \sum_{s=1}^{T}(T-s+1) a(s) u(s) \\
& \leq \frac{\varepsilon}{1-\alpha \eta} \sum_{s=1}^{T}(T-s+1) a(s)\|u\| \\
& \leq\|u\| .
\end{aligned}
$$

Thus $\|A u\| \leq\|u\|, u \in K \cap \partial \Omega_{1}$. 
Further, since $f_{\infty}=\infty$, there exists $L_{2}>0$ such that $f(u) \geq \rho u$, for $u \geq L_{2}$, where $\rho>0$ is chosen so that

$$
\frac{\rho \gamma}{1-\alpha \eta} \sum_{s=\eta}^{T}(T-s+1) a(s) \geq 1 .
$$

Let $L=\max \left\{2 L_{1}, \frac{L_{2}}{\gamma}\right\}$ and $\Omega_{2}=\left\{u \in \mathbb{R}^{T+2}, u(t) \geq 0\right.$ for all $\left.t \in \mathbb{N}_{T+1},\|u\|<L\right\}$. Then $u \in K \cap \partial \Omega_{2}$ implies

$$
\min _{t \in \mathbb{N}_{T+1}} u(t) \geq \gamma\|u\|=\gamma L \geq L_{2}
$$

and so

$$
\begin{aligned}
& A u(\eta) \\
= & \frac{1}{1-\alpha \eta} \sum_{s=1}^{T}(T-s+1) a(s) f(u(s)) \\
& -\frac{\alpha}{2(1-\alpha \eta)} \sum_{s=1}^{\eta-1}(\eta-s)(\eta-s+1) a(s) f(u(s))-\sum_{s=1}^{\eta-1}(\eta-s) a(s) f(u(s)) \\
= & \frac{1}{1-\alpha \eta} \sum_{s=\eta}^{T}(T-s+1) a(s) f(u(s))+\frac{1}{1-\alpha \eta} \sum_{s=1}^{\eta-1}(T-s+1) a(s) f(u(s)) \\
& -\frac{\alpha}{2(1-\alpha \eta)} \sum_{s=1}^{\eta-1}\left(\eta^{2}+\eta-2 \eta s+s^{2}-s\right) a(s) f(u(s))-\sum_{s=1}^{\eta-1}(\eta-s) a(s) f(u(s)) \\
= & \frac{1}{1-\alpha \eta} \sum_{s=\eta}^{T}(T-s+1) a(s) f(u(s))+\frac{2(T-\eta)+2-\alpha \eta}{2(1-\alpha \eta)} \sum_{s=1}^{\eta-1} a(s) f(u(s)) \\
& +\frac{\alpha}{2(1-\alpha \eta)} \sum_{s=1}^{\eta-1}(\eta(\eta-s)+s) a(s) f(u(s))+\frac{\alpha}{2(1-\alpha \eta)} \sum_{s=1}^{\eta-1} s(\eta-s) a(s) f(u(s)) .
\end{aligned}
$$

Hence

$$
\begin{aligned}
A u(\eta) & \geq \frac{1}{1-\alpha \eta} \sum_{s=\eta}^{T}(T-s+1) a(s) f(u(s)) \\
& \geq \frac{\rho}{1-\alpha \eta} \sum_{s=\eta}^{T}(T-s+1) a(s) u(s) \\
& \geq \frac{\rho \gamma}{1-\alpha \eta} \sum_{s=\eta}^{T}(T-s+1) a(s)\|u\| \\
& \geq\|u\| .
\end{aligned}
$$


Hence, $\|A u\| \geq\|u\|, u \in K \cap \partial \Omega_{2}$. Bay the first part of Theorem 1.1, $A$ has a fixed point in $K \cap\left(\bar{\Omega}_{2} \backslash \Omega_{1}\right)$ such that $L_{1} \leq\|u\| \leq L$.

(ii) Sublinear case: $f_{0}=\infty$ and $f_{\infty}=0$. Since $f_{0}=\infty$, we may choose $L_{3}>0$ so that $f(u) \geq M u$, for $0<u \leq L_{3}$, where $M>0$ satisfies

$$
\frac{M \gamma}{1-\alpha \eta} \sum_{s=\eta}^{T}(T-s+1) a(s) \geq 1 .
$$

Thus, if we let

$$
\Omega_{3}=\left\{u \in \mathbb{R}^{T+2}, u(t) \geq 0 \text { for all } t \in \mathbb{N}_{T+1},\|u\|<L_{3}\right\},
$$

then for $u \in K \cap \partial \Omega_{3}$, we get

$$
\begin{aligned}
A u(\eta)= & \frac{1}{1-\alpha \eta} \sum_{s=1}^{T}(T-s+1) a(s) f(u(s)) \\
& -\frac{\alpha}{2(1-\alpha \eta)} \sum_{s=1}^{\eta-1}(\eta-s)(\eta-s+1) a(s) f(u(s))-\sum_{s=1}^{\eta-1}(\eta-s) a(s) f(u(s)) \\
\geq & \frac{1}{1-\alpha \eta} \sum_{s=\eta}^{T}(T-s+1) a(s) f(u(s)) \\
\geq & \frac{M}{1-\alpha \eta} \sum_{s=\eta}^{T}(T-s+1) a(s) u(s) \\
\geq & \frac{M \gamma}{1-\alpha \eta} \sum_{s=\eta}^{T}(T-s+1) a(s)\|u\| \geq\|u\| .
\end{aligned}
$$

Thus, $\|A u\| \geq\|u\|, u \in K \cap \partial \Omega_{3}$. Now, since $f_{\infty}=0$, there exists $L_{4}>0$ such that $f(u) \leq \lambda u$, for $u \geq L_{4}$, where $\lambda>0$ satisfies

$$
\frac{\lambda}{1-\alpha \eta} \sum_{s=1}^{T}(T-s+1) a(s) \leq 1 .
$$

Choose $L^{\prime}=\max \left\{2 L_{3}, \frac{L_{4}}{\gamma}\right\}$. Let

$$
\Omega_{4}=\left\{u \in \mathbb{R}^{T+2}, u(t) \geq 0 \text { for all } t \in \mathbb{N}_{T+1},\|u\|<L^{\prime}\right\} .
$$

Then $u \in K \cap \partial \Omega_{4}$ implies

$$
\inf _{t \in \mathbb{N}_{T+1}} u(t) \geq \gamma\|u\|=\gamma L^{\prime} \geq L_{4} .
$$


Therefore,

$$
\begin{aligned}
A u(t) & \leq \frac{1}{1-\alpha \eta} \sum_{s=1}^{T}(T-s+1) a(s) f(u(s)) \\
& \leq \frac{\lambda}{1-\alpha \eta} \sum_{s=1}^{T}(T-s+1) a(s) u(s) \\
& \leq \frac{\lambda}{1-\alpha \eta} \sum_{s=1}^{T}(T-s+1) a(s)\|u\| \\
& \leq\|u\| .
\end{aligned}
$$

Thus $\|A u\| \leq\|u\|, u \in K \cap \partial \Omega_{4}$. By the second part of Theorem 1.1, $A$ has a fixed point in $K \cap\left(\bar{\Omega}_{4} \backslash \Omega_{3}\right)$ such that $L_{3} \leq\|u\| \leq L^{\prime}$. This completes the sublinear part of the theorem. Therefore, the problem (1.1) has at least one positive solution.

\section{Some Examples}

In this section, in order to illustrate our results, we consider some examples.

Example 4.1. Consider the BVP

$$
\begin{cases}\triangle^{2} u(t-1)+t e^{3 t}(1+\sin (u))=0, & t \in \mathbb{N}_{1,5} \\ \triangle u(0)=0, & u(6)=\frac{2}{7} \sum_{s=1}^{3} u(s) .\end{cases}
$$

Set $\alpha=\frac{2}{7}, \eta=3, T=5, a(t)=t e^{3 t}, f(u)=1+\sin (u)$.

We can show that

$$
0<\alpha=\frac{2}{7}<\frac{1}{3}=\frac{1}{\eta} .
$$

A simple calculation we get $f_{0}=\infty, f_{\infty}=0$ and (ii) of Theorem 3.1 holds. Then BVP (4.1) has at least one positive solution.

Example 4.2. Consider the BVP

$$
\begin{cases}\triangle^{2} u(t-1)+\left(3 t^{2}+2 t+1\right)\left(u^{2} \ln (u+1)\right)=0, & t \in \mathbb{N}_{1,7} \\ \triangle u(0)=0, & u(8)=\frac{3}{17} \sum_{s=1}^{5} u(s) .\end{cases}
$$

Set $\alpha=\frac{3}{17}, \eta=5, T=7, a(t)=3 t^{2}+2 t+1, f(u)=u^{2} \ln (u+1)$.

We can show that

$$
0<\alpha=\frac{3}{17}<\frac{1}{5}=\frac{1}{\eta}
$$

A simple calculation we get $f_{0}=0, f_{\infty}=\infty$ and (i) of Theorem 3.1 holds. Then BVP (4.2) has at least one positive solution. 
Acknowledgements. The authors gratefully acknowledge the reviewers for their helpful comments.

\section{REFERENCES}

[1] R. P. Agarwal, Focal Boundary Value Problems for Differential and Difference Equations, Kluwer Academic Publishers, Dordrecht, 1998.

[2] R. P. Agarwal, D. O'Regan and P. J. Y. Wong, Positive Solutions of Differential, Difference and Integral Equations, Kluwer Academic Publishers, Dordrecht, 1999.

[3] Z. Bai, X. Liang and Z. Du, Triple positive solutions for some second-order boundary value problem on a measure chain, Comput. Math. Appl. 53 (2007), 1832-1839.

[4] S. Chasreechat and T. Sitthiwirattham, On a summation boundary value problem for a secondorder difference equations with resonance, J. Comput. Anal. Appl. 22(2) (2017), 298-309.

[5] C. P. Gupta, Solvability of a three-point nonlinear boundary value problem for a second order ordinary differential equations, J. Math. Anal. Appl. 168(2) (1992), 540-551.

[6] X. He and W. Ge, Existence of three solutions for a quasilinear two-point boundary value problem, Comput. Math. Appl. 45 (2003), 765-769.

[7] J. Henderson and H. B. Thompson, Existence of multiple solutions for second order discrete boundary value problems, Comput. Math. Appl. 43 (2002), 1239-1248.

[8] V. A. Ilin and E. I. Moiseev, Nonlocal boundary-value problem of the first kind for a SturmLiouville operator in its differential and finite difference aspects, J. Differential Equations 23 (1987), 803-810.

[9] M. A. Krasnoselskii, Positive Solutions of Operator Equations, P. Noordhoof, Gronignen, 1964.

[10] R. W. Leggett and L. R. Williams, Multiple positive fixed points of nonlinear operators on ordered Banach spaces, Indiana Univ. Math. J. 28 (1979), 673-688.

[11] X. Lin and W. Liu, Three positive solutions of a second order difference equations with three-point boundary value problem, J. Appl. Math. Comut. 31 (2009), 279-288.

[12] B. Liu, Positive solutions of a nonlinear three-point boundary value problem, Appl. Math. Comput. 132 (2002), 11-28.

[13] J. Reunsumrit and T. Sitthiwirattham, Positive solutions of difference summation boundary value problems for a second-order difference equation, J. Comput. Anal. Appl. 19(2) (2015), 319-334.

[14] T. Sitthiwirattham and S. Ratanapun, Positive solutions of a second-order difference equation with summation boundary value problem, Int. Journal of Math. Analysis 7(2) (2013), 91-103.

[15] T. Sitthiwirattham and J. Tariboon, Positive solutions to a generalized second order difference equation with summation boundary value problem, Journal of Applied Mathematics 2012 (2012), Article ID 569313.

[16] J. Tariboon and T. Sitthiwirattham, Positive solutions of a nonlinear three-point integral boundary value problem, Boundary Value Problems 2010 (2010), Article ID 519210.

[17] G. Zhang and R. Medina, Three-point boundary value problems for difference equations, Comp. Math. Appl. 48 (2004), 1791-1799.

\footnotetext{
${ }^{2}$ Department of MAThematics And InFormatics, University of Souk Ahras, P.O. Box 1553, Souk Ahras, Algeria

E-mail address: abd_ardjouni@yahoo.fr
} 
${ }^{1}$ Department of Mathematics, UniVERSITY OF ANNABA,

P.O. Box 12, Annaba, Algeria

E-mail address: fyb1500@gmail.com, adjoudi@yahoo.com 